\title{
Estimates for Solutions of Differential Equations in a Banach Space via Commutators
}

https://doi.org/10.1515/msds-2018-0001

Received May 24, 2017; accepted January 26, 2018

Abstract: In a Banach space we consider the equation $d x(t) / d t=(A+B(t)) x(t)(t \geq 0)$, where $A$ is a constant bounded operator, and $B(t)$ is a bounded variable operator. Norm estimates for the solutions of the considered equation are derived in terms of the commutator $A B(t)-B(t) A$. These estimates give us sharp stability conditions. Our results are new even in the finite dimensional case. We also discuss applications of the obtained results to a class of integro-differential equations.

Keywords: Banach space, differential equation, solution estimates, stability, integro-differential equation

MSC: 34G10, 34D05, 34D20

\section{Introduction and statement of the main result}

Let $X$ be a Banach space with a norm $\|\cdot\|$ and the unit operator $I$. By $\mathcal{B}(X)$ we denote the algebra of bounded linear operators in $X$. For a $C \in \mathcal{B}(X), \sigma(C)$ is the spectrum, $\|C\|$ is the operator norm. We consider the equation

$$
\frac{d y(t)}{d t}=(A+B(t)) y(t) \quad(t \geq 0)
$$

where $A \in \mathcal{B}(X)$ is a constant operator and $B(t)$ is a function with values in $\mathcal{B}(X)$ uniformly bounded on $[0, \infty)$ and Riemann-integrable on each finite segment. A solution to (1.1) for given $y_{0} \in X$ is a function $y:[0, \infty) \rightarrow X$ having at each point $t \geq 0$ a strong derivative and satisfying (1.1) for all $t \geq 0$ and $y(0)=y_{0}$. The existence and uniqueness of solutions under considerations is obvious.

Equation (1.1) is said to be exponentially stable, if there are positive constants $M, \epsilon$, such that $\|y(t)\| \leq$ $M \exp [-\epsilon t]\|y(0)\|(t \geq 0)$ for any solution of (1.1).

Equation (1.1) can be considered as the equation

$$
\frac{d x(t)}{d t}=C(t) x(t)
$$

with a variable linear operator $C(t)$. This identification which is a common device in the theory of differential equations when passing from a given equation to an abstract evolution equation turns out to be useful also here. Observe that $C(t)$ in the considered case has a special form: it is the sum of operators $A$ and $B(t)$. This fact allows us to use the information about the coefficients more completely than the theory of differential equations (1.2) containing an arbitrary operator $C(t)$.

The basic method for the stability analysis of (1.2) is the direct Lyapunov method [5]. By that method many very strong results are obtained, but finding Lyapunov's functions is often connected with serious mathematical difficulties. Norm estimates following from the multiplicative representations of solutions, such as the Wintner inequality [5, Theorem III.3.7], give us explicit stability conditions, provided $C(t)$ is a dissipative operator (for more details see Section 3 below). In addition, in [14] a stability test for (1.2) has been derived

\footnotetext{
^Corresponding Author: Michael Gil’: Department of Mathematics, Ben Gurion University of the Negev, P.0. Box 653, Beer-
} Sheva 84105, Israel, E-mail: gilmi@bezeqint.net 
for equations in a Hilbert space whose operator coefficients have "small" derivatives. The approach in [14] is an extension of the freezing method for ordinary differential equations.

Let $X(t, s)$ be evolution operator of (1.1) and $U(t, s)(t \geq s \geq 0)$ the evolution operator of the equation

$$
\dot{y}(t)=B(t) y(t) \quad(t \geq 0) .
$$

In this paper, we suggest estimates for the sup-norm of solutions to (1.1) via the commutator $K(t):=[A, B(t)]=$ $A B(t)-B(t) A$. These estimates give us sharp stability conditions, provided we have estimates for $U(t, s)$. Besides, we do not require that the operator in (1.2) is dissipative. To the best of our knowledge, our results are new even in finite dimensional case, $\mathrm{cf}$. [18]. We also discuss applications of the obtained results to a class of integro-differential equations. The theory of stability of various classes of integro-differential equations is rather rich, cf. [2, 4], [7]-[10], [16, 20, 21] and references therein, but stability conditions via commutators have not been investigated.

Put

$$
Y(t, s)=e^{A(t-s)} U(t, s), \kappa=\sup _{t \geq 0}\|K(t)\| \text { and }\|X\|_{C}:=\sup _{t \geq s \geq 0}\|X(t, s)\| .
$$

It is assumed that there are a positive constant $c_{0}$ and a real one $b_{0}$, such that

$$
\|U(t, s)\| \leq c_{0} \exp \left[b_{0}(t-s)\right](t \geq s \geq 0) .
$$

We say that an $A \in \mathcal{B}(X)$ is stable if its spectrum is in the open left half-plane. Now we are in a position to formulate our main result.

Theorem 1.1. Let condition (1.4) hold and the operator $A+b_{0} I$ be stable. In addition, let

$$
\zeta:=\kappa c_{0}\left(\int_{0}^{\infty}\left\|e^{\left(A+b_{0} I\right) t}\right\| d t\right)^{2}<1 .
$$

Then

$$
\|X-Y\|_{C}:=\sup _{t \geq s \geq 0}\|X(t, s)-Y(t, s)\| \leq \frac{\zeta\|Y\|_{C}}{1-\zeta}
$$

and equation (1.1) is exponentially stable.

The proof of this theorem is presented in the next section. Note that under consideration

$$
\|Y\|_{C} \leq c_{0} \sup _{t}\left\|e^{\left(A+b_{0} I\right) t}\right\| .
$$

Theorem 1.1 is sharp: if $A$ commutes with $B(t)$, then $X(t, s)=Y(t, s)(t \geq s)$. From (1.6) it directly follows

$$
\|X\|_{C} \leq \frac{\|Y\|_{C}}{1-\zeta} .
$$

\section{Proof of Theorem 1.1}

Let $\left[e^{A t}, B(t)\right]:=e^{t A} B(t)-B(t) e^{A t}$.

Lemma 2.1. One has

$$
\left[e^{A(t-s)}, B(t)\right]=\int_{s}^{t} e^{(v-s) A} K(t) e^{(t-v) A} d v \quad(0 \leq s \leq t<\infty) .
$$

Proof. We have

$$
\int_{S}^{t} e^{(v-s) A} K(t) e^{(t-v) A} d v=\int_{s}^{t} e^{(v-s) A}(A B(t)-B(t) A) e^{(t-v) A} d v=
$$




$$
\begin{gathered}
\int_{s}^{t}\left(\left(\frac{d}{d v} e^{(v-s) A}\right) B(t) e^{(t-v) A}+e^{(v-s) A} B(t)\left(\frac{d}{d v} e^{(t-v) A}\right)\right) d v= \\
\int_{s}^{t} \frac{d}{d v}\left(e^{(v-s) A} B(t) e^{(t-v) A}\right) d v=\left.e^{(v-s) A} B(t) e^{(t-v) A}\right|_{v=s} ^{t}=e^{A(t-s)} B(t)-B(t) e^{A(t-s)} .
\end{gathered}
$$

We thus get the required result.

The previous lemma is a generalization of Lemma 2.1 from [15].

Lemma 2.2. With the notation $F(t, s):=\left[e^{A(t-s)}, B(t)\right] U(t, s)$, let

$$
\gamma(F):=\sup _{s} \int_{s}^{\infty}\|F(t, s)\| d t<1 .
$$

Then

$$
\|X\|_{C} \leq \frac{\|Y\|_{C}}{1-\gamma(F)}
$$

and

$$
\|X-Y\|_{C} \leq \frac{\gamma(F)\|Y\|_{C}}{1-\gamma(F)} .
$$

Proof. We have

$$
\begin{aligned}
& \frac{\partial}{\partial t} Y(t, s)=\frac{\partial}{\partial t}\left(e^{A(t-s)} U(t, s)\right)=\left(\frac{\partial}{\partial t} e^{A(t-s)}\right) U(t, s)+e^{A(t-s)} \frac{\partial}{\partial t} U(t, s) \\
= & A e^{A(t-s)} U(t, s)+e^{A(t-s)} B(t) U(t, s)=(A+B(t)) e^{A(t-s)} U(t, s)+F(t, s) .
\end{aligned}
$$

Thus,

$$
\partial Y(t, s) / \partial t=(A+B(t)) Y(t, s)+F(t, s) .
$$

Subtracting (1.1) from (2.4), for a fixed $s$, we get

$$
\frac{\partial}{\partial t}(Y(t, s)-X(t, s))=(A+B(t))(Y(t, s)-X(t, s))+F(t, s) .
$$

Since $Y(s, s)=X(s, s)=I$, we can write

$$
Y(t, s)-X(t, s)=\int_{s}^{t} X\left(t, t_{1}\right) F\left(t_{1}, s\right) d t_{1} .
$$

Consequently,

$$
\|Y(t, s)-X(t, s)\| \leq \int_{0}^{t}\left\|X\left(t, t_{1}\right)\right\|\left\|F\left(t_{1}, s\right)\right\| d t_{1},
$$

and therefore

$$
\|X(t, s)\| \leq\|Y(t, s)\|+\int_{s}^{t}\left\|X\left(t, t_{1}\right)\right\|\left\|F\left(t_{1}, s\right)\right\| d t_{1} .
$$

Hence, for any finite $t>0$ we obtain

$$
\sup _{0 \leq s \leq v \leq t}\|X(v, s)\| \leq\|Y\|_{C}+\sup _{0 \leq s \leq v \leq t}\|X(v, s)\| \gamma(F)
$$

Now (2.1) implies

$$
\sup _{0 \leq s \leq v \leq t}\|X(v, s)\| \leq\|Y\|_{C} /(1-\gamma(F)) .
$$

This proves (2.2). From (2.6) and (2.2), inequality (2.3) follows. This proves the lemma. 
Proof of Theorem 1.1: By Lemma 2.1,

$$
\|F(t, s)\| \leq\left\|\left[e^{A(t-s)}, B(t)\right]\right\|\|U(t, s)\| \leq \kappa\|U(t, s)\| \int_{s}^{t}\left\|e^{A(v-s)}\right\|\left\|e^{A(t-v)}\right\| d v .
$$

Hence,

$$
\int_{s}^{a}\|F(t, s)\| d t \leq \hat{\gamma}(s, a)(a>s)
$$

where

$$
\hat{\gamma}(s, a):=\kappa \int_{s}^{a}\|U(t, s)\| \int_{s}^{t}\left\|e^{A(v-s)}\right\|\left\|e^{A(t-v)}\right\| d v d t .
$$

So $\gamma(F) \leq \sup _{s} \hat{\gamma}(s, \infty)$. From (1.4) for a finite $a>s$ it follows

$$
\begin{gathered}
\hat{\gamma}(s, a) \leq \kappa c_{0} \int_{s}^{a} e^{b_{0}(t-s)} \int_{s}^{t}\left\|e^{A(t-v)}\right\|\left\|e^{A(v-s)}\right\| d v d t \\
=\kappa c_{0} \int_{s}^{a} \int_{s}^{t} e^{b_{0}(t-v)} e^{b_{0}(v-s)}\left\|e^{A(t-v)}\right\|\left\|e^{A(v-s)}\right\| d v d t \\
\leq \kappa c_{0} \int_{s}^{a}\left\|e^{\left(A+b_{0} I\right)(t-s)}\right\| d t \int_{s}^{a}\left\|e^{\left(A+b_{0} I\right)(v-s)}\right\| d v .
\end{gathered}
$$

Thus

$$
\gamma(F) \leq \kappa c_{0}\left\|e^{\left(A+b_{0} I\right) t}\right\|_{L^{1}(0, \infty)}^{2}
$$

Here

$$
\left\|e^{\left(A+b_{0} I\right) t}\right\|_{L^{1}(0, \infty)}=\int_{0}^{\infty}\left\|e^{\left(A+b_{0} I\right) t}\right\| d t .
$$

Now Lemma 2.2 proves (1.6) and (1.7). Inequality (1.7) means that (1.1) is Lyapunov stable. Furthermore, substitute

$$
y(t)=u_{\epsilon}(t) e^{-\epsilon t}(\epsilon>0)
$$

into (1.1). Then

$$
d u_{\epsilon}(t) / d t=(A+B(t)+\epsilon I) u_{\epsilon}(t) .
$$

Applying our above arguments to (2.10) one can assert that equation (2.10) with small enough $\epsilon>0$ is Lyapunov stable. So due to (2.9) equation (1.1) is exponentially stable. This proves the theorem.

\section{Auxiliary results}

To apply Theorem 1.1 to integro-differential equations we need some auxiliary results presented in this section. Introduce the products

$$
\begin{gathered}
\prod_{1 \leq k \leq m}^{\leftarrow}\left(I+B\left(t_{k}^{(m)}\right) \delta_{k}\right):= \\
\left(I+B\left(t_{m}^{(m)}\right) \delta_{m}\right)\left(I+B\left(t_{m-1}^{(m)}\right) \delta_{m-1}\right) \cdots\left(I+B\left(t_{1}^{(m)}\right) \delta_{1}\right)
\end{gathered}
$$

where

$$
s=t_{1}^{(m)}<t_{2}^{(m)}<\ldots<t_{m}^{(m)}=t \text { and } \delta_{k}=t_{k}^{(m)}-t_{k-1}^{(m)}(k=1, \ldots, m)
$$


I.e., the arrow over the symbol of the product means that the indexes of the co-factors increase from right to left. The strong limit of these products as $\max _{k} \delta_{k} \rightarrow 0$ (if it exists) is called the left multiplicative integral and is denoted by $\int_{[s, t]}^{\leftarrow}(I+B(s) d s)$. As it is well-known,

$$
U(t, s)=\int_{[s, t]}^{\overleftarrow{ }}(I+B(s) d s)
$$

This equality is proved in [6]; in the finite dimensional case it is proved in [11, Chapter XV, Section 6] but for operators in a Banach space the proof is the same.

Furthermore, introduce the products

$$
\begin{gathered}
\prod_{1 \leq k \leq m}^{\overleftarrow{ }} e^{B\left(t_{k}^{(m)}\right) \delta_{k}}:=e^{B\left(t_{m}^{(m)}\right) \delta_{m}} e^{B\left(t_{m-1}^{(m)}\right) \delta_{m-1}} \ldots e^{B\left(t_{1}^{(m)}\right) \delta_{1}} \\
\left(s=t_{1}^{(m)}<t_{2}^{(m)}<\ldots<t_{m}^{(m)}=t\right) .
\end{gathered}
$$

The strong limit of these products as $\max _{k} \delta_{k} \rightarrow 0$ (if it exists) will be called the left exponentially multiplicative integral and denoted by $\int_{[s, t]}^{\leftarrow} e^{B(t) d t}$. As it is shown in [5, Section III.1],

$$
U(t, s)=\overleftarrow{\int_{[s, t]}} e^{B(t) d t}
$$

Lemma 3.1. Let there be a real function $\phi(t)$ Riemann-integrable on each finite segment, such that

$$
\left\|e^{B(t) \delta}\right\| \leq e^{\phi(t) \delta}(t \geq 0)
$$

for all sufficiently small $\delta>0$. Then

$$
\|U(t, s)\| \leq \exp \left[\int_{s}^{t} \phi\left(s_{1}\right) d s_{1}\right](t \geq s \geq 0) .
$$

Proof. Condition (3.3) implies

$$
\left\|\prod_{1 \leq k \leq m}^{\overleftarrow{ }} e^{\left.B\left(t_{k}^{(m)}\right)\right) \delta_{k}}\right\| \leq \prod_{k=1}^{m} e^{\phi\left(t_{k}^{(m)}\right) \delta_{k}}=\exp \left[\sum_{k=1}^{m} \phi\left(t_{k}^{(m)}\right) \delta_{k}\right] .
$$

The passage to the limit as $m \rightarrow \infty$ and representation (3.2) give the required estimate.

Similarly, applying representation (3.1), we obtain the following result.

Lemma 3.2. Let there be a real Riemann-integrable function function $\phi(t)$, such that

$$
\|I+A(t) \delta\| \leq 1+\phi(t) \delta(t \geq 0)
$$

for all sufficiently small $\delta>0$. Then inequality (3.4) is valid.

Let $X$ be a Hilbert space. Put $\Lambda(B(s))=\sup \sigma(\Re B(s))$, where $\Re B(s)=\frac{1}{2}\left(B(s)+B^{\star}(s)\right)$ and the asterisk means the adjointness. Taking in (3.5) $\phi(t)=\Lambda(B(t))$, we arrive at the Wintner inequality

$$
\|U(t, s)\| \leq \exp \left[\int_{s}^{t} \Lambda\left(B\left(s_{1}\right)\right) d s_{1}\right](t \geq s \geq 0) .
$$

Theorem 1.1, and Lemmas 3.1 and 3.2 imply

Corollary 3.3. Assume that one of conditions (3.3) or (3.5) holds and

$$
b_{0}: \inf _{t \geq s \geq 0} \frac{1}{(t-s)} \int_{s}^{t} \phi\left(t_{1}\right) d t_{1}<\infty .
$$

Then $\|U(t, s)\| \leq \exp \left[b_{0}(t-s)\right](t \geq s \geq 0)$ and equation (1.1) is exponentially stable, provided

$$
\kappa\left\|e^{\left(A+b_{0} I\right) t}\right\|_{L^{1}(0, \infty)}^{2}<1 .
$$




\section{Integro-differential equations}

Put $\Omega=[0,1] \times[0,1]$. In this section $X=L^{2}(\Omega)$ is the Hilbert space of complex square integrable functions defined on $\Omega$ with the traditional scalar product and norm. Consider the equation

$$
\begin{gathered}
\frac{\partial u(t, x, y)}{\partial t}=c(t, x, y) u(t, x, y)+\int_{0}^{x} \psi_{1}(x, s) u(t, s, y) d s+\int_{0}^{1} \psi_{2}\left(t, y, s_{1}\right) u\left(t, x, s_{1}\right) d s_{1} \\
(0 \leq x, y \leq 1 ; t \geq 0),
\end{gathered}
$$

where $c(\cdot, \cdot, \cdot):[0, \infty) \times \Omega \rightarrow \mathbb{C}$ is piece-wise continuous, $\psi_{1}(\cdot, \cdot): \Omega \rightarrow \mathbb{C} \psi_{2}(\cdot, \cdot, \cdot):[0, \infty) \times \Omega \rightarrow \mathbb{C}$ are given functions satisfying the conditions pointed below. Equations of the type (4.1) play an essential role in numerous applications, in particular, in kinetic theory [3], transport theory [17], continuous mechanics [1], the dynamics of populations [19], etc. Define the operators $A$ and $B(t)$ by

$$
(A w)(x, y)=\int_{0}^{x} \psi_{1}(x, s) w(s, y) d s
$$

and

$$
(B(t) w)(x, y)=c(t, x, y) w(x, y)+\int_{0}^{1} \psi_{2}\left(t, y, s_{1}\right) w\left(x, s_{1}\right) d s_{1} \quad\left(x, y \in[0,1] ; w \in L^{2}(\Omega)\right),
$$

respectively. Under consideration we have

$$
(K(t) w)(x, y)=\int_{0}^{x} m(t, x, y, s) w(s, y) d s,
$$

where $m(t, x, y, s)=\psi_{1}(x, s)(c(t, s, y)-c(t, x, y))$. Assume that

$$
N_{2}(A)=\left(\int_{0}^{1} \int_{0}^{x}\left|\psi_{1}(x, s)\right|^{2} d s d x\right)^{1 / 2}<\infty
$$

and $\psi_{2}$ provides the boundedness of the operator $M(t)$ defined on $L^{2}(0,1)$ by

$$
(M(t) v)(y)=\int_{0}^{1} \psi_{2}(t, y, s) v(s) d s \quad\left(y \in[0,1] ; v \in L^{2}(0,1)\right)
$$

Obviously, $\Lambda(B(t)) \leq \sup _{x, y} \Re c(t, x, y)+\Lambda(M(t))$. Various estimates for the eigenvalues of partial integral operators can be found in [13]. Assume that $\Lambda(B(t))<0$ and (3.7) holds with $\phi(t)=\Lambda(B(t))$ and $b_{0}<0$. Furthermore, under consideration $A$ is a quasi-nilpotent (Volterra) operator. According to [12, Example 7.10.3], we have

$$
\left\|e^{\left(A+b_{0} I\right) t}\right\| \leq e^{b_{0} t} \sum_{k=0}^{\infty} \frac{t^{k} N_{2}^{k}(A)}{(k !)^{3 / 2}}(t \geq 0) .
$$

So $\left\|e^{\left(A+b_{0} I\right) t}\right\|_{L^{1}(0, \infty)} \leq J\left(b_{0}, A\right)$, where

$$
J\left(b_{0}, A\right)=\sum_{k=0}^{\infty} \frac{N_{2}^{k}(A)}{\left|b_{0}\right|^{k+1}(k !)^{1 / 2}} .
$$

Making use of Corollary 3.3, we can assert that equation (4.1) is exponentially stable, provided $\kappa J^{2}\left(b_{0}, A\right)<1$. 


\section{References}

[1] V.M. Aleksandrov and E.V. Kovalenko, Problems in Continuous Mechanics with Mixed Boundary Conditions, Nauka, Moscow 1986. In Russian.

[2] J.A.D. Appleby and D.W. Reynolds, On the non-exponential convergence of asymptotically stable solutions of linear scalar Volterra integro-differential equations, Journal of Integral Equations and Applications, 14, no 2 (2002), 521-543.

[3] M.C. Cercignani, Mathematical Methods in Kinetic Theory, Macmillian, New York, 1969.

[4] Chuhu Jin and Jiaowan Luo, Stability of an integro-differential equation, Computers and Mathematics with Applications, 57 (2009) 1080-1088.

[5] Yu L. Daleckii, and M. G. Krein, Stability of Solutions of Differential Equations in Banach Space, Amer. Math. Soc., Providence, R. I. 1974.

[6] J.D. Dollard and Ch. N. Friedman, Product Integration with Applications to Differential Equations. Encyclopedia of Mathematics and its applications; v.10., London, Addison-Wesley Publ. Company, 1979.

[7] A. Domoshnitsky and Ya. Goltser, An approach to study bifurcations and stability of integro-differential equations. Math. Comput. Modelling, 36 (2002), 663-678.

[8] A.D. Drozdov, Explicit stability conditions for integro-differential equations with periodic coefficients, Math. Methods Appl. Sci. 21 (1998), 565-588.

[9] A.D. Drozdov and M. I. Gil', Stability of linear integro-differential equations with periodic coefficients. Quart. Appl. Math. 54 (1996), 609-624.

[10] N.T. Dung, On exponential stability of linear Levin-Nohel integro-differential equations, Journal of Mathematical Physics 56, 022702 (2015); doi: 10.1063/1.4906811

[11] F.R. Gantmakher, Theory of Matrices. Nauka, Moscow, 1967. In Russian.

[12] M.I. Gil', Operator Functions and Localization of Spectra, Lecture Notes In Mathematics vol. 1830, Springer-Verlag, Berlin, 2003.

[13] M.I. Gil', Spectrum and resolvent of a partial integral operator. Applicable Analysis, 87, no. 5, (2008) 555-566.

[14] M.I. Gil', On stability of linear Barbashin type integro-differential equations, Mathematical Problems in Engineering, 2015, Article ID 962565, (2015), 5 pages.

[15] M.I. Gil', A bound for the Hilbert-Schmidt norm of generalized commutators of nonself-adjoint operators, Operators and Matrices, 11, no. 1 (2017), 115-123

[16] Ya. Goltser and A. Domoshnitsky, Bifurcation and stability of integrodifferential equations, Nonlinear Anal. 47 (2001), $953-$ 967.

[17] H.G. Kaper, C.G. Lekkerkerker, and J. Hejtmanek, Spectral Methods in Linear Transport Theory, Birkhauser, Basel, 1982.

[18] W.J. Rugh, Linear System Theory. Prentice Hall, Upper Saddle River, New Jersey, 1996.

[19] H. R. Thieme, A differential-integral equation modelling the dynamics of populations with a rank structure, Lect. Notes Biomath. 68 (1986), 496-511

[20] J. Vanualailai and S. Nakagiri, Stability of a system of Volterra integro-differential equations J. Math. Anal. Appl. 281 (2003) 602-619

[21] B. Zhang, Construction of Liapunov functionals for linear Volterra integrodifferential equations and stability of delay systems, Electron. J. Qual. Theory Differ. Equ. 30 (2000) 1-17. 